\title{
Activation of the FAK/PI3K pathway is crucial for AURKA-induced epithelial-mesenchymal transition in laryngeal cancer
}

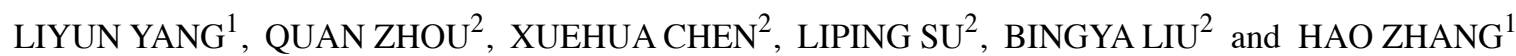 \\ ${ }^{1}$ Department of Otolaryngology and ${ }^{2}$ Shanghai Key Laboratory of Gastric Neoplasms, \\ Shanghai Institute of Digestive Surgery, Ruijin Hospital, School of Medicine, \\ Shanghai Jiaotong University, Shanghai 200025, P.R. China
}

Received January 25, 2016; Accepted March 21, 2016

DOI: $10.3892 /$ or.2016.4872

\begin{abstract}
Laryngeal squamous cell carcinoma (LSCC) is one of the most common malignant tumors, and the main cause of death is metastasis. Overexpression of aurora kinase A (AURKA) plays an important role in the metastasis of LSCC. However, the mechanism by which AURKA promotes the metastasis of LSCC is poorly understood. Recent accumulating evidence indicates that epithelial-mesenchymal transition (EMT) may be one of the mechanisms of tumor metastasis. In the present study, we studied whether AURKA may induce EMT to promote the metastasis of LSCC. CCK-8 and plate colony-formation assays were carried out to show that AURKA significantly promoted the proliferation of Hep2 cells. Immunofluorescence staining and western blotting showed that EMT-related proteins changed in a time-dependent manner along with the alteration of AURKA, with decreased expression of $\mathrm{N}$-cadherin, vimentin and slug and increased expression of E-cadherin. Additionally, downregulation of the expression of AURKA inhibited FAK/PI3K pathway activity. Inhibition of the FAK/PI3K pathway caused less mesenchymal-like characteristics and reduced the mobility, migration and invasion of Hep2 cells. In conclusion, AURKA may induce EMT to promote metastasis via activation of the FAK/PI3K pathway in LSCC. Those regulatory factors may present new diagnostic biomarkers and potential therapeutic targets for LSCC.
\end{abstract}

Correspondence to: Dr Hao Zhang, Department of Otolaryngology, Ruijin Hospital, School of Medicine, Shanghai Jiaotong University, 197 Ruijin 2nd Road, Shanghai 200025, P.R. China

E-mail: zhanghaoent@163.com

Abbreviations: LSCC, laryngeal squamous cell carcinoma; HNSCC, head and neck squamous cell carcinoma; AURKA, aurora kinase A; EMT, epithelial-mesenchymal transition

Key words: laryngeal cancer, AURKA, epithelial-mesenchymal transition, FAK/P13K pathway, metastasis

\section{Introduction}

Laryngeal squamous cell carcinoma (LSCC), arises from the larynx epithelium and is one of the most common head and neck squamous cell carcinomas (HNSCCs) with a high rate of metastasis and a poor prognosis $(1,2)$. Currently, LSCC accounts for almost $2 \%$ of all malignancies worldwide $(3,4)$. Although recent treatment outcomes are improved, patients still have a poor prognosis with a 5-year survival rate of $\sim 60 \%$ (5). The main cause of death is cancer metastasis (6) and the intrinsic microenvironment of tumor cells has an important role in tumor metastasis (7). Therefore, a better understanding of the underlying molecular metastasis-related mechanisms of LSCC is essential for developing effective therapeutic strategies to improve the survival rate and quality of life of these patients.

Aurora kinase A (AURKA), a homologue of aurora/Ipl1related kinase, is located at chromosome $20 \mathrm{q} 13.2$ (8). It is an oncogene that causes various epithelial malignant tumors, including pancreatic (9), ovarian (10), colorectal (11) and prostate cancer (12). We previously reported that the expression of AURKA was elevated in human LSCC and promoted the metastasis of LSCC $(13,14)$. However, the underlying mechanism by which AURKA enhances the metastasis of LSCC is still poorly understood.

Recent accumulating evidence indicates that the occurrence of epithelial-mesenchymal transition (EMT) may be the principal mechanism of cancer metastasis, including bladder (15), colorectal (16), pancreatic (17), breast (18), oral (19), thyroid (20) and prostate cancer (21), and LSCC (22). EMT is the process by which epithelial cells de-differentiate into mesenchymal-like derivatives (23). During the process, epithelial cells acquire the characteristics of mesenchymal cells, such as lack of polarization, increased motility and invasion, decreased cell-cell junctions and chemotherapeutic resistance (24-26). Recently, D'Assoro et al reported that AURKA induced EMT to promote distant metastasis in breast cancer (27). This indicates an association between AURKA and EMT and cancer metastasis.

Therefore, in the present study, we investigated whether AURKA is involved in EMT to promote the metastasis of 
LSCC. The epithelial-related protein E-cadherin and the mesenchymal-related proteins, $\mathrm{N}$-cadherin, vimentin and slug, were investigated either in AURKA-downregulated or parental Hep2 cells. Effects of inhibition of the FAK/PI3K pathway on the mesenchymal-like characteristics and cellular mobility, migration and invasion ability were also studied in these Hep2 cells.

\section{Materials and methods}

Cell cultures. The human laryngeal cancer Hep2 cell line (Hep2 cells) was obtained from Shanghai Institutes of Biological Sciences, Chinese Academy of Sciences. Hep2 cells were cultured in Dulbecco's modified Eagle's medium (DMEM) with 10\% fetal bovine serum (FBS) (both from Gibco, Bedford, MA, USA) and $1 \%$ penicillin/streptomycin at $37^{\circ} \mathrm{C}$ under $5 \% \mathrm{CO}_{2}$.

Cell proliferation assay. Cell proliferation was measured with the CCK-8 assay kit (Gibco). Briefly, a total density of $3 \times 10^{3} \mathrm{Hep} 2$ cells/well, growing in logarithmic phase, in $100 \mu \mathrm{l}$ of DMEM were plated into the 96-well plates. Then, $10 \mu \mathrm{l}$ of CCK-8 was added into every well at the specific time points. After culture for $2 \mathrm{~h}$ at $37^{\circ} \mathrm{C}$ under $5 \% \mathrm{CO}_{2}, \mathrm{OD}_{450}$ absorbance values were monitored. In the present study, the proliferation of T-Hep2 cells following treatment with the selective inhibitor of AURKA (VX680) (Selleck Chemicals, Houston, TX, USA), which was dissolved in dimethyl sulfoxide (DMSO), was measured at 0,24 and $48 \mathrm{~h}$ for 2 days.

Plate colony-formation assay. Hep2 cells treated with VX680 for $48 \mathrm{~h}$ were the experimental group and the cells treated with DMSO or without any treatment were considered the controls. Cells at a density of $2 \times 10^{3}$ were layered into 6-well plates. After culture for 2 weeks with DMEM containing $10 \%$ FBS, the cells were washed with phosphatebuffered saline (PBS) 3 times and fixed with crystal violet for $30 \mathrm{~min}$ at room temperature. The formed colonies were counted in every well.

Immunofluorescence staining. Hep2 cells treated with VX680 for $48 \mathrm{~h}$ or without any treatment, at the density of $5 \times 10^{4}$, were seeded into Millicell ${ }^{\circledR}$ EZ Slides (Millipore, Bedford, MA, USA). The cells were fixed with $4 \%$ paraformaldehyde (PFA) for $30 \mathrm{~min}$ when they adhered to the wall of the slides. Then, the slides were rinsed 3 times with $1 \mathrm{X}$ PBS for 5 min each time, followed by blocking with primary cocktail (Cell Signaling Technology, Danvers, MA, USA) (diluted 1:100 as indicated on the datasheet in antibody dilution buffer) to examine the expression of EMT markers, vimentin and E-cadherin. Following overnight incubation at $4{ }^{\circ} \mathrm{C}$, the blocking solution was aspirated and the slides were washed 3 times with 1X PBS for 5 min each time and were then incubated with Alexa Fluor ${ }^{\circledR} 555$ and Alexa Flour ${ }^{\circledR} 488$ (Cell Signaling Technology) (diluted 1:100 as indicated on the datasheet in antibody dilution buffer) for $1 \mathrm{~h}$ at room temperature in the dark. Then, the slides were rinsed 3 times with $1 \mathrm{X}$ PBS for $5 \mathrm{~min}$ each. For immunofluorescence staining of p-AURKA (anti-p-AURKA; 1:100; Cell Signaling Technology), blocking with 5\% BSA in PBS containing $0.05 \%$ Triton for $1 \mathrm{~h}$ at room temperature was the only difference in the protocol. Vimentin and E-cadherin were analyzed using fluorescence microscopy magnification, x10 (U-ULS100HG; Olympus Optical Co., Ltd., Tokyo, Japan) (magnification, $\mathrm{x} 10$ ).

Western blot analysis. Hep2 cells, treated with VX680, the inhibitor of FAK (TAE226), and the inhibitor of PI3K (omipalisib) (both from Selleck Chemicals), respectively, were lysed with RIPA buffer containing $1 \%$ protease inhibitor cocktail (100:1) and quantified by the BCA protein assay kit (all from Pierce, Rockford, IL, USA) according to the manufacturer's instructions. Harvested proteins were separated with $10 \%$ sodium dodecyl sulfate-polyacrylamide gel electrophoresis (SDS-PAGE) for $2 \mathrm{~h}$ and transferred onto polyvinylidene difluoride (PVDF) membranes (Millipore) for $2 \mathrm{~h}$. Then, the membranes were blocked with $5 \%$ non-fat milk dissolved in 1X TBST $(150 \mathrm{mM} \mathrm{NaCl}, 0.05 \%$ Tween20, $10 \mathrm{mM}$ Tris-HCl, $\mathrm{pH}$ 8.0) for $2 \mathrm{~h}$, and incubated with the primary antibodies at $4^{\circ} \mathrm{C}$ overnight including anti-E-cadherin $(1: 2,000)$, anti-N-cadherin $(1: 2,000)$, anti-vimentin $(1: 2,000)$, anti-slug $(1: 2,000)$ (all from Proteintech Group, Inc., Rosemont, IL, USA), anti-p-AURKA $(1: 2,000)$, anti-AURKA $(1: 2,000)$, anti-FAK (1:2,000), anti-p-FAK (Tyr397, 1:2,000) (all from Cell Signaling Technology), anti-PI3K (1:2,000), anti-p-PI3K $(1: 2,000)$ and GAPDH $(1: 5,000)$ (all from Abcam). After overnight incubation, the PVDF membranes were washed with 1X TBST 3 times and incubated with a secondary antibody (1:5,000; Cell Signaling Technology) for $2 \mathrm{~h}$ at room temperature. Lastly, the membranes were detected with an enhanced chemiluminescence detection system (Amersham Biosciences, Piscataway, NJ, USA).

Wound healing assay. Hep2 cells treated with TAE226 and omipalisib were considered the experimental groups, and groups treated with DMSO or without any treatment were the control groups. Hep2 cells at a density of $1 \times 10^{6}$ cell/well were seeded into 6-well plates. After overnight culture, the Hep2 cells were scratched with $20-\mu 1$ pipette tips to form straight lines. The floating cells were removed using 1X PBS 3 times. Finally, the wound lines were photographed at 0,24 and $48 \mathrm{~h}$ at a magnification of $\mathrm{x} 2$.

Cell migration and invasion assays. A density of $2 \times 10^{5}$ cell/well treated with TAE226, omipalisib, DMSO or without any treatment suspended in $200 \mu \mathrm{l}$ of serum-free DMEM were placed into the top of Transwell chambers (Corning, Tewksbury, MA, USA) and the lower chambers were filled with $600 \mu 1$ of DMEM with $10 \%$ FBS. After overnight culture, non-migratory Hep2 cells were removed with a cotton swab from the top surface of the chamber and the lower filter of the chamber was stained with crystal violet solution for $30 \mathrm{~min}$ at $37^{\circ} \mathrm{C}$. Then, the migratory Hep2 cells were photographed using microscopy at magnification, $\mathrm{x} 20$. Briefly, the experimental produces of the cell invasion and the cell migration assays were identical except that BD Matrigel ${ }^{\mathrm{TM}}$ (Becton-Dickinson Labware, Bedford, MA, USA) covered the membrane of the top chamber.

Statistical analysis. Data of all experiments were analyzed by GraphPad Prism 6 software (GraphPad, San Diego, CA, 




B

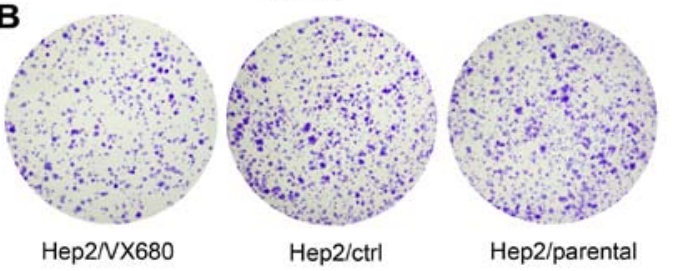

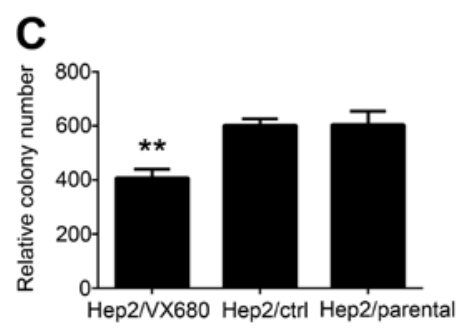

Figure 1. Downregulation of AURKA by VX680 reduces the proliferation of Hep2 cells. (A) Effects of VX680 on cell proliferation were analyzed by CCK-8 assay. VX680 markedly suppressed Hep2 cell proliferation $\left({ }^{*} \mathrm{P}<0.05\right)$. (B) Effects of VX680 on cell proliferation were analyzed by plate colony formation assay. VX680 significantly inhibited Hep2 cell proliferation. (C) Quantification of the relative colony number $\left({ }^{* *} \mathrm{P}<0.01\right)$.

A
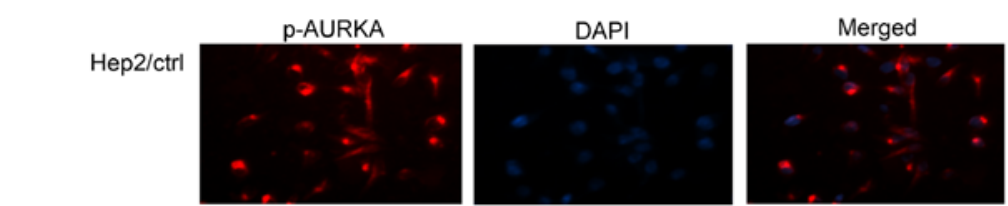

Hep2/VX680/48
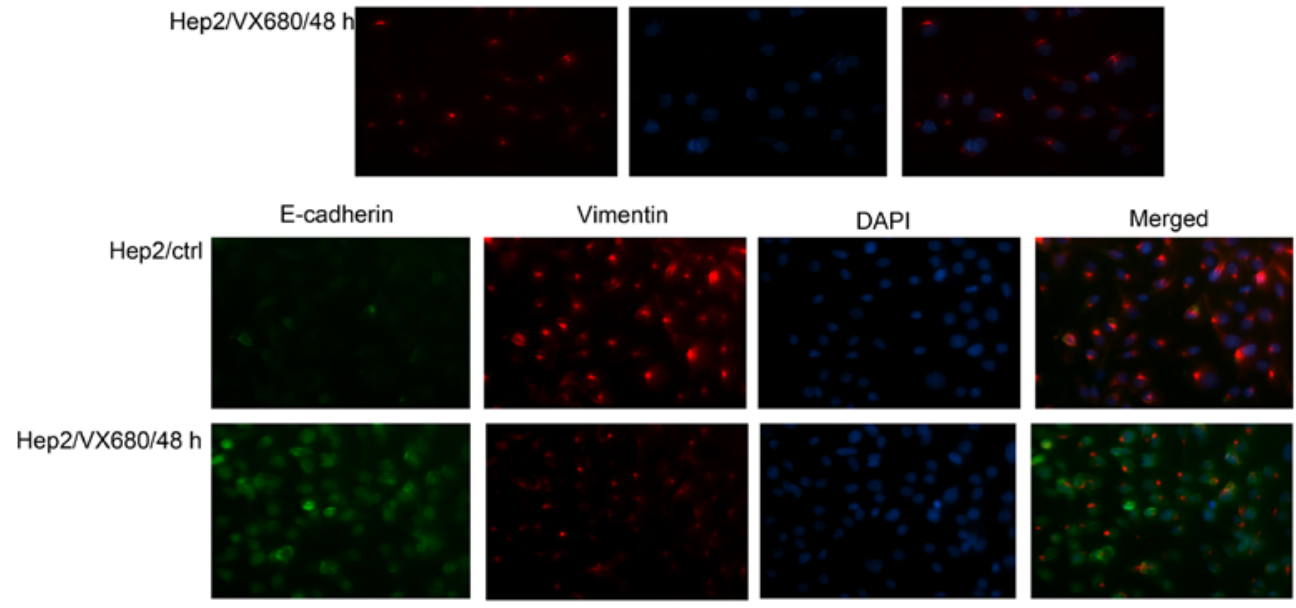

B
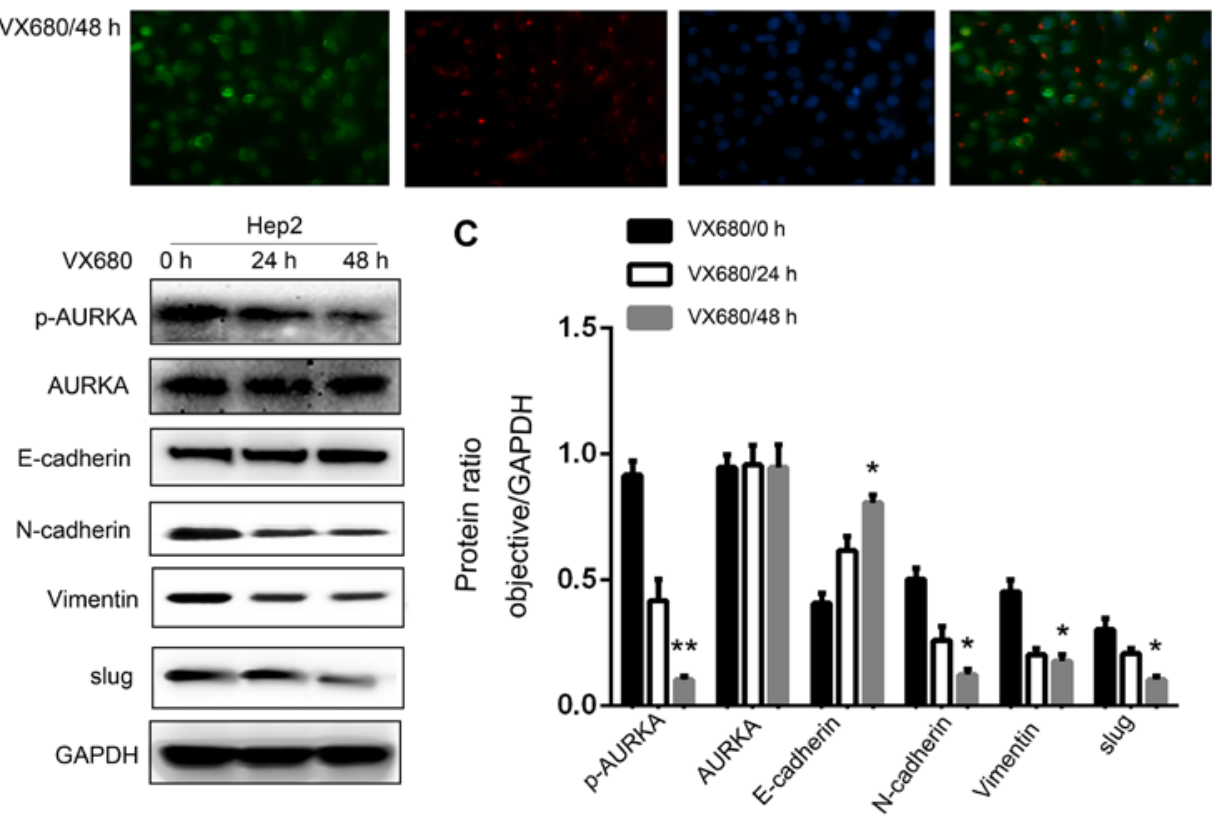

Figure 2. Downregulation of AURKA using VX680 induces less mesenchymal-like characteristics in Hep2 cells. (A) Effects of VX680 on the expression of EMT-related proteins were analyzed by immunofluorescence staining. Hep2 cells treated with VX680 showed low expression of p-AURKA and vimentin, while high expression of E-cadherin. (B) Effects of VX680 on the expression of related proteins were analyzed by western blotting. Expression of p-AURKA, $\mathrm{N}$-cadherin, vimentin and slug was decreased, while that of E-cadherin was increased. (C) Quantification of the protein ratio $\left({ }^{*} \mathrm{P}<0.05,{ }^{* *} \mathrm{P}<0.01\right)$.

USA) and are shown as mean \pm SD. The Student's t-test was performed to assess the differences between experimental groups and controls. The level of significance was set at $\mathrm{P}<0.05$, and a high level of significance was set at $\mathrm{P}<0.01$.

\section{Results}

Inhibition of AURKA with VX680 reduces the proliferation of Hep 2 cells. Our previous study showed that AURKA was 

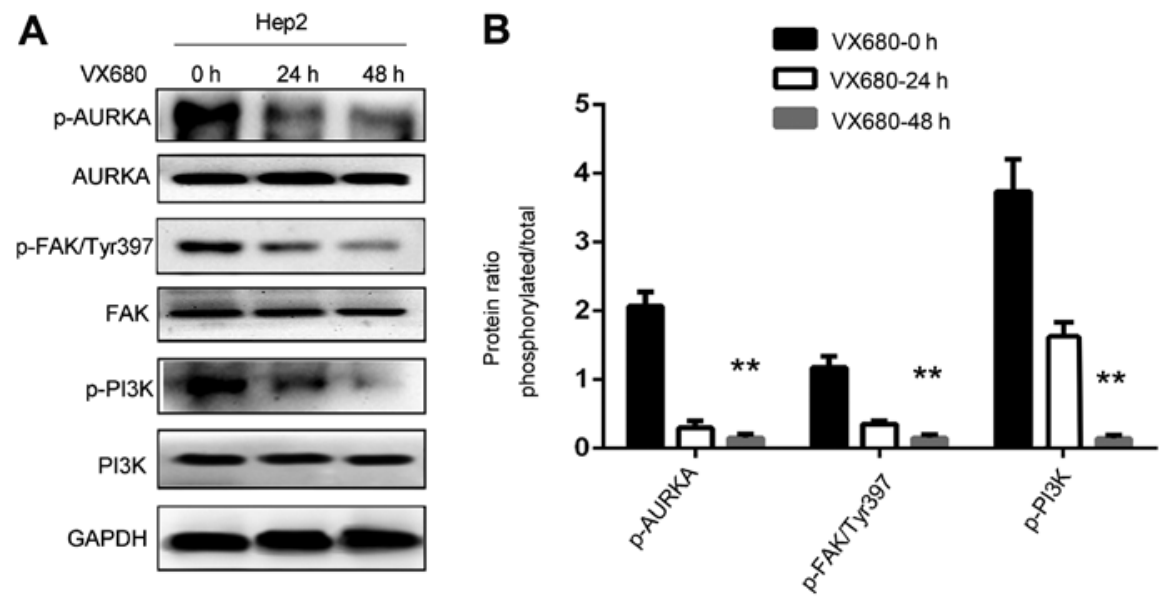

Figure 3. Downregulation of AURKA using VX680 inhibits the phosphorylation of the FAK/PI3K pathway in Hep2 cells. (A) Effects of VX680 on the expression of related proteins were analyzed by western blotting. Expression of p-AURKA, p-FAK (Tyr397) and p-PI3K was reduced. (B) Quantification of the protein ratio $\left({ }^{* *} \mathrm{P}<0.01\right)$.

overexpressed in Hep2 cells and promoted the metastasis of LSCC (13,14). We downregulated AURKA with VX680 $(80 \mathrm{~nm} / \mathrm{ml})(28)$, a selective inhibitor of AURKA, in the Hep2 cells for 24 and $48 \mathrm{~h}$ (Hep2/VX680). Hep2 cells treated with DMSO (Hep2/ctrl) and without any treatment (Hep2/parental) were the control groups. The results of the CCK- 8 assay showed less proliferation in the Hep2/VX680 cells compared with the Hep2/ctrl and Hep2/parental cells $(\mathrm{P}<0.05$; Fig. 1A). The plate colony-formation assay showed parallel results in the Hep2/VX680 $(404 \pm 18.95)$, Hep2/ctrl $(601 \pm 16.74)$ and Hep2/parental cells $(607 \pm 24.11)(\mathrm{P}<0.01$; Fig. $1 \mathrm{~B}$ and $\mathrm{C})$. These results indicated that AURKA significantly promoted cell proliferation and that VX680 effectively reduced the function of AURKA.

Downregulation of AURKA causes less mesenchymal-like characteristics in Hep2 cells. To investigate whether AURKA is involved in EMT to promote the metastasis of LSCC, the EMT-related proteins E-cadherin, N-cadherin, vimentin and slug were assessed in the Hep2 cells treated with VX680 at specific times. The results of immunofluorescence staining showed that the expression of p-AURKA and vimentin in the Hep2 cells was lower, while the expression of E-cadherin was higher when compared with these levels in the control group (Fig. 2A). Subsequently, the results of western blotting also revealed that the expression of p-AURKA was decreased almost 3 -fold ( $\mathrm{P}<0.01$; Fig. 2B and $\mathrm{C})$ and the EMT-related protein levels were altered in a time-dependent manner along with the alteration of AURKA. Expression of E-cadherin was increased and expression of $\mathrm{N}$-cadherin, vimentin and slug was decreased (Fig. 2B and C). All of the aforementioned observations indicated that AURKA may induce EMT to promote the metastasis of LSCC.

AURKA promotes the metastasis of LSCC by enhancing phosphorylation of the FAK/PI3K pathway. Given that the FAK pathway is regarded as a regulatory factor in tumor metastasis (29-31) and FAK is involved in PI3K-promoted EMT in lung cancer (32), we aimed to demonstrate that AURKA regulates those factors to induce EMT. As expected, the expression of p-FAK (Tyr397) and p-PI3K was reduced following the diminution of p-AURKA in the Hep2 cells $(\mathrm{P}<0.01$; Fig. $3 \mathrm{~A}$ and $\mathrm{B})$, which demonstrated that FAK and PI3K were regulated by AURKA and played a key role in contributing to the tumorigenesis of LSCC.

Inhibition of the FAK/PI3K pathway causes less mesenchymal-like characteristics. To explore whether the FAK/PI3K pathway plays a key role in the induction of EMT in LSCC, we utilized the FAK inhibitor TAE226 $(2.1 \mu \mathrm{M} / \mathrm{ml})(33)$, and PI3K inhibitor omipalisib $(500 \mathrm{~nm} / \mathrm{ml})(34)$ dissolved in DMSO. Treatment with TAE226 altered the EMT-related proteins in a time-dependent manner. The level of E-cadherin was increased, while $\mathrm{N}$-cadherin, vimentin and slug were decreased, indicating that FAK caused more mesenchymallike characteristics (Fig. 4A and B). p-AURKA, AURKA, p-FAK (Tyr397), FAK, p-PI3K and PI3K in the Hep2/TAE226 cells were also examined. The expression of p-FAK (Tyr397) was nearly decreased 2-fold after treatment with TAE226 for $24 \mathrm{~h}(\mathrm{P}<0.05$; Fig. $4 \mathrm{C}$ and $\mathrm{D})$. In addition, the expression of p-PI3K was decreased $(\mathrm{P}<0.01$; Fig. $4 \mathrm{C}$ and $\mathrm{D})$, whereas p-AURKA, AURKA, FAK and PI3K were not changed, indicating that FAK is a downstream factor of AURKA.

EMT-related proteins were also inhibited in the Hep2/omipalisib cells. These observations showed that E-cadherin was increased, while $\mathrm{N}$-cadherin, vimentin and slug were decreased, which also demonstrated that inhibition of PI3K caused less mesenchymal-like characteristics (Fig. 4E and F). Moreover, p-AURKA, AURKA, p-FAK (Tyr397), FAK, p-PI3K and PI3K were assessed after using omipalisib for 0,6 and $12 \mathrm{~h}$. From the results of the western blotting, p-PI3K was markedly decreased ( $\mathrm{P}<0.01$; Fig. $4 \mathrm{G}$ and $\mathrm{H})$, whereas p-AURKA, AURKA, p-FAK (Tyr397), FAK and $\mathrm{PI} 3 \mathrm{~K}$ were not changed, which illustratied that PI3K is a downstream factor of AURKA/FAK (Fig. 4G and H).

Inhibition of the FAK/PI3K pathway decreases cellular mobility, migration and invasion. To further demonstrate that activation of the FAK/PI3K pathway may be crucial for AURKA-induced EMT in LSCC metastasis, wound healing, 
A

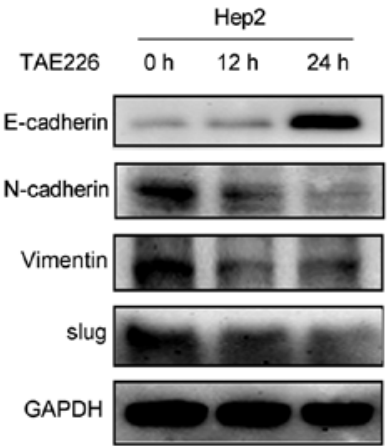

C

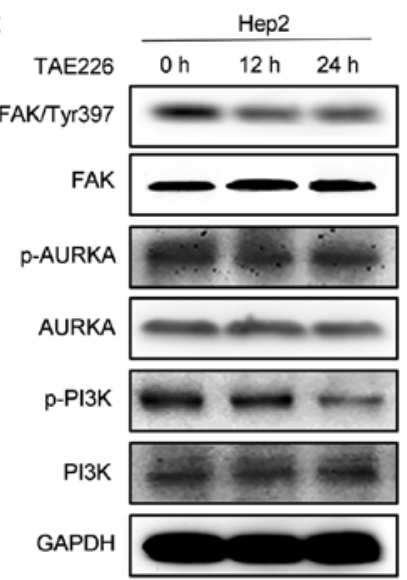

E

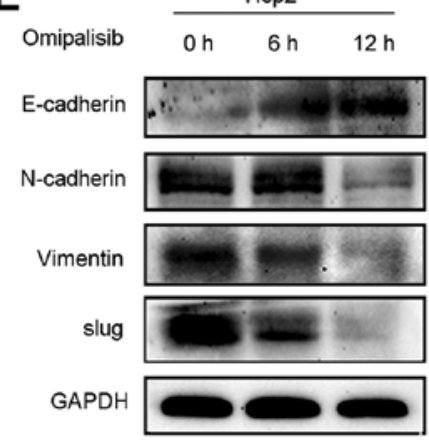

G



B

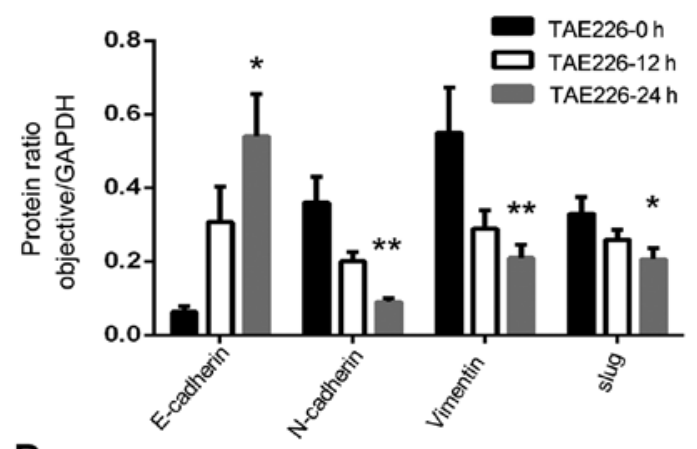

D

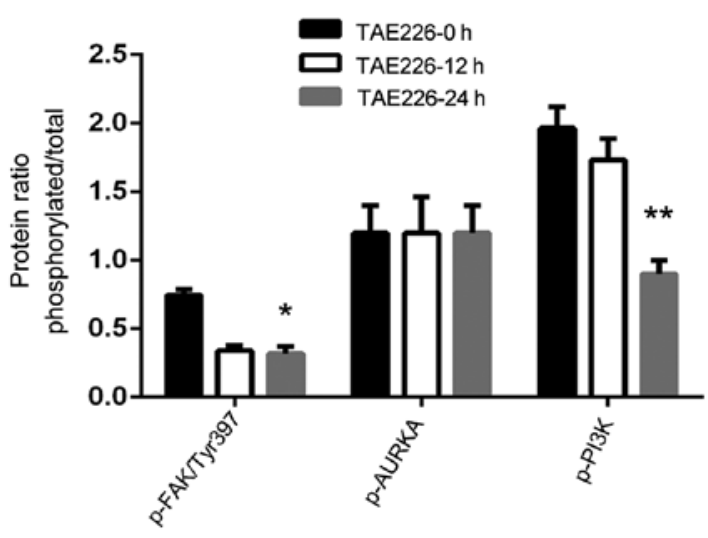

$\mathbf{F}$



H

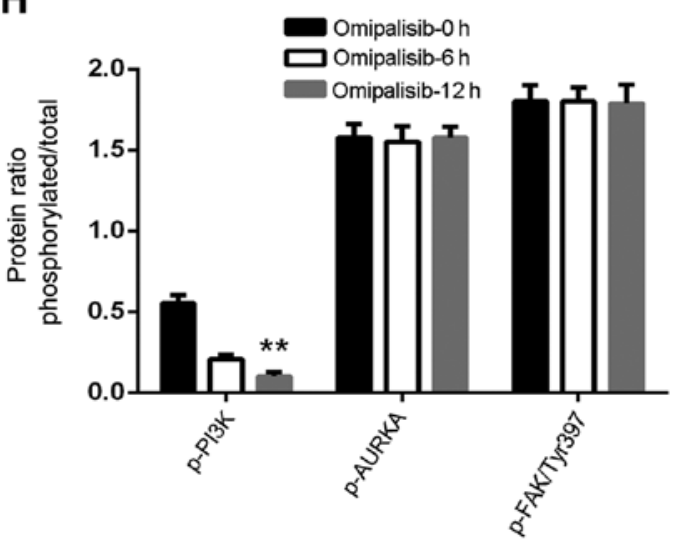

Figure 4. Effects of the inhibition of FAK/PI3K using TAE226 or omipalisib in Hep2 cells. (A) Effects of TAE226 on the expression of EMT-related proteins were analyzed by western blotting. The expression of E-cadherin was increased, whereas N-cadherin, vimentin and slug were decreased. (B) Quantification of EMT-related protein ratio after treatment with TAE226 ( $\left.{ }^{*} \mathrm{P}<0.05,{ }^{* *} \mathrm{P}<0.01\right)$. (C) Effects of TAE226 on the expression of p-AURKA, AURKA, p-FAK (Tyr397), FAK, p-PI3K and PI3K were analyzed by western blotting. Expression of p-FAK (Tyr397) and p-PI3K was decreased, while the others were not changed. (D) Quantification of the protein ratio after treatment with TAE226 ("P $\left.<0.05,{ }^{* *} \mathrm{P}<0.01\right)$. (E) Effects of omipalisib on the expression of EMT-related proteins as analyzed by western blotting. Expression of E-cadherin was increased, whereas N-cadherin, vimentin and slug were decreased. (F) Quantification of EMTrelated protein ratio after treatment with omipalisib $\left({ }^{*} \mathrm{P}<0.05,{ }^{* *} \mathrm{P}<0.01\right)$. (G) Effects of omipalisib on the expression of the regulatory factors as analyzed by western blotting. Only p-PI3K was decreased. (H) Quantification of related protein ratio after treatment with omipalisib $\left({ }^{* *} \mathrm{P}<0.01\right)$. 

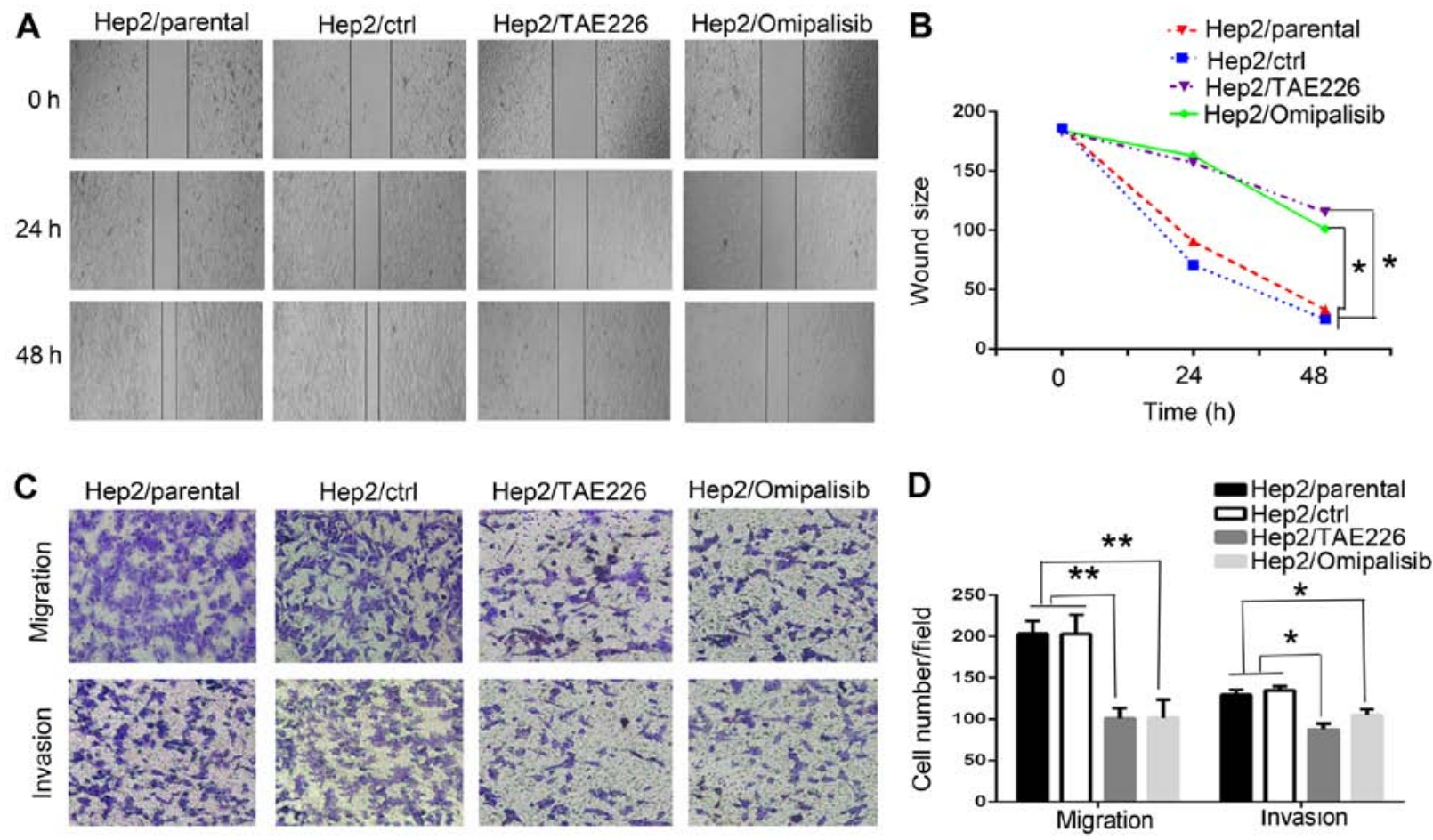

Figure 5. Inhibition of the FAK/PI3K pathway by TAE226/omipalisib reduces mobility, migration and invasion in Hep2 cells. (A) Effects of TAE226/omipalisib on cell mobility in the wound healing assay. FAK/PI3K promoted Hep2 cell mobility. (B) Quantification of wound size ("P<0.05). (C) Effects of TAE226/ omipalisib on cell migration and invasion by migration and invasion assays. FAK/PI3K promoted the migration and invasion of Hep2 cells. (D) Quantification of cell number/field $\left({ }^{*} \mathrm{P}<0.05,{ }^{* *} \mathrm{P}<0.01\right)$.

migration and invasion assays were performed. We downregulated FAK and PI3K with TAE226 (Hep2/TAE226) and omipalisib (Hep2/omipalisib), respectively. Similarly, Hep2 cells treated with DMSO (Hep2/ctrl) and without any treatment (Hep2/parental) were considered the control groups. First the wound was scratched and observed for 0,24 and $48 \mathrm{~h}$. The results showed that Hep2/TAE226 and Hep2/omipalisib cells moved slightly, while Hep2/ctrl and Hep2/parental cells almost reached the middle of the scratch, indicating that FAK and PI3K caused more mesenchymal-like characteristics and markedly promoted Hep2 cell mobility ( $\mathrm{P}<0.05$; Fig. $5 \mathrm{~A}$ and $\mathrm{B})$.

Similarly, the results of the migration assay indicated that the numbers of Hep2/TAE226 (101 \pm 7.23$)$ and Hep2/omipalisib cells $(101 \pm 12.7)$ migrating through the Transwell chamber were significantly less than these numbers in the Hep2/parental (203 \pm 8.76$)$ and Hep2/ctrl cells $(203 \pm 13.33)$. The invasion assay also showed parallel results with Hep2/TAE226 $(88 \pm 4.10)$, Hep2/omipalisib (105 \pm 3.93$)$, Hep2/parental (130 \pm 3.46$)$, Hep2/ctrl cells (135 \pm 2.89 ) (Fig. 5C and D). These observations indirectly illustrated that FAK and PI3K acquired more mesenchymal-like characteristics and increased mobility, migration and invasion.

\section{Discussion}

Cancer metastasis accounts for the majority of tumor-related deaths worldwide. Understanding the potential mechanism of metastasis has been long recognized as an essential target in the treatment of cancer. However, the precise molecular and cellular metastasis-related mechanisms are poorly understood (35). Based on our previous research showing that
AURKA plays a key role in the tumorigenesis and metastasis of LSCC in vitro (13) and in vivo (14), as well as growing evidence suggesting that EMT may be the principal mechanism of cancer metastasis (15-22), we explored the correlation between AURKA and EMT in contributing to the metastasis of LSCC. To the best of our knowledge, no previous study has been reported dealing with this domain in LSCC.

AURKA, a member of the evolutionarily conserved aurora serine/threonine kinase family, controls cell cycle (36) by centrosome maturation, mitotic entry, centrosome separation, bipolar spindle assembly, chromosome alignment, cytokinesis and mitotic exit (37). Dysfunctional regulation during mitosis leads to genetic instability and tumorigenesis. In the present study, results of the CCK-8 and plate colony-formation assays showed that VX680 inhibited the proliferation of Hep2 cells, and that AURKA is a tumor-inducing gene in LSCC. Notably, mesenchymal-related proteins, $\mathrm{N}$-cadherin, vimentin and slug, which endow cells with more migratory and invasive properties (26), were decreased in a time-dependent manner following the reduction of AURKA, while the epithelialrelated protein E-cadherin was negatively correlated with AURKA in the Hep2 cells. According to the aforementioned observations, we demonstrated that AURKA may promote the metastasis of LSCC by inducing EMT and may be regarded as a mesenchymal marker.

In addition, the process of metastasis involves numerous potential signaling pathways. Niu et al stated that EMT could be correlated with the $\mathrm{p} 38 \mathrm{MAPK} / \mathrm{PI} 3 \mathrm{~K} / \mathrm{Akt} / \mathrm{mTOR}$ pathway to promote osteosarcoma metastasis (36), Kuo et al indicated that EMT involves the NF- $\mathrm{kB} / \mathrm{ZEB} 1$ pathway to promote lung cancer cell migration and invasion (38). The FAK-mediated 
PI3K/AKT pathway acts through EMT to enhance lung cancer metastasis (31). In the present study, down regulation of the expression of AURKA reduced the expression of FAK and PI3K indicating that AURKA may act on FAK and PI3K to facilitate tumor metastasis. Subsequently, TAE226 was applied to block the expression of p-FAK (Tyr397). Notably, the expression of p-PI3K was also decreased, whereas p-AURKA, AURKA, FAK and PI3K were not altered, indicating that FAK is the downstream factor of AURKA. p-PI3K was markedly decreased after treatment with omipalisib for $12 \mathrm{~h}$; inversely p-AURKA, AURKA, p-FAK (Tyr397), FAK and PI3K were not altered, indicating that PI3K is a downstream factor of AURKA/FAK. Finally, inhibition of FAK or PI3K altered the expression of EMT markers which was the same as the treatment of VX680 and allowed mesenchymal cells to acquire the characteristics of epithelial cells to reduce mobility, migration and invasion in Hep2 cells. All of these results imply that the FAK/PI3K pathway is under the regulation of AURKA to promote the process of EMT and enhance the metastasis of LSCC.

The present study suggests that VX680, TAE226 and omipalisib, which are inhibitors and implicated in the AURKA/FAK/PI3K signaling pathway, should not only be considered for single use but also for combination therapy in the treatment of patients with LSCC. Certainly, various doses and schedules of targeted therapeutic drugs should be examined in clinical trials to ensure the effectiveness and safety of LSCC therapy. Taken together, our results demonstrated that AURKA may enhance metastasis by inducing EMT via the FAK/PI3K pathway in LSCC. In addition, in light of our observations of the therapeutic potential in laryngeal cancer, the AURKA/FAK/PI3K pathway may present new diagnostic biomarkers and potential therapeutic targets for LSCC.

\section{Acknowledgements}

The present study was supported by grants from the Science and Technology Commission of Shanghai Municipality (no. 12ZR1418700) and the Clinical Science and Technology Innovation Project of Shenkang Hospital Development Center (SHDC12015114).

\section{References}

1. Li D, Feng J, Wu T, Wang Y, Sun Y, Ren J and Liu M: Long intergenic noncoding RNA HOTAIR is overexpressed and regulates PTEN methylation in laryngeal squamous cell carcinoma. Am J Pathol 182: 64-70, 2013.

2. Bingol F, Yoruk O, Bingol BO, Erdemci B, Ozkan O and Mazlumoglu MR: Estimation of the efficacy of chemo-radiotherapy on tumor regression in the patients with laryngeal cancer via computerized tomography using the Cavalieri method. Acta Otolaryngol 136: 164-167, 2016.

3. Halec G, Holzinger D, Schmitt M, Flechtenmacher C, Dyckhoff G, Lloveras B, Höfler D, Bosch FX and Pawlita M: Biological evidence for a causal role of HPV16 in a small fraction of laryngeal squamous cell carcinoma. Br J Cancer 109: 172-183, 2013.

4. Yu GP, Mehta V, Branovan D, Huang Q, Hashibe M, Zhang ZF and Schantz SP: Improved survival among patients with base of tongue and tonsil cancer in the United States. Cancer Causes Control 23: 153-164, 2012.

5. Shen Z, Li Q, Deng H, Lu D, Song H and Guo J: Long noncoding RNA profiling in laryngeal squamous cell carcinoma and its clinical significance: Potential biomarkers for LSCC. PLoS One 9: e108237, 2014.
6. Mitra A, Mishra L and Li S: EMT, CTCs and CSCs in tumor relapse and drug-resistance. Oncotarget 6: 10697-10711, 2015.

7. Amend SR and Pienta KJ: Ecology meets cancer biology: The cancer swamp promotes the lethal cancer phenotype. Oncotarget 6: 9669-9678, 2015.

8. Marumoto T, Zhang D and Saya H: Aurora-A - a guardian of poles. Nat Rev Cancer 5: 42-50, 2005.

9. Rojanala S, Han H, Muñoz RM, Browne W, Nagle R, Von Hoff DD and Bearss DJ: The mitotic serine threonine kinase, Aurora-2, is a potential target for drug development in human pancreatic cancer. Mol Cancer Ther 3: 451-457, 2004.

10. Watanabe T, Imoto I, Katahira T, Hirasawa A, Ishiwata I, Emi M, Takayama M, Sato A and Inazawa J: Differentially regulated genes as putative targets of amplifications at $20 \mathrm{q}$ in ovarian cancers. Jpn J Cancer Res 93: 1114-1122, 2002.

11. Bischoff JR, Anderson L, Zhu Y, Mossie K, Ng L, Souza B, Schryver B, Flanagan P, Clairvoyant F, Ginther C, et al: A homologue of Drosophila aurora kinase is oncogenic and amplified in human colorectal cancers. EMBO J 17: 3052-3065, 1998.

12. Xing Z, Gao S, Duan Y, Han H, Li L, Yang Y and Li Q: Delivery of DNAzyme targeting aurora kinase $A$ to inhibit the proliferation and migration of human prostate cancer. Int J Nanomedicine 10: 5715-5727, 2015

13. Zhang H, Chen X, Jin Y, Liu B and Zhou L: Overexpression of Aurora-A promotes laryngeal cancer progression by enhancing invasive ability and chromosomal instability. Eur Arch Otorhinolaryngol 269: 607-614, 2012.

14. Zhang H, Chen X, Liu B and Zhou L: Effects of stable knockdown of Aurora kinase A on proliferation, migration, chromosomal instability, and expression of focal adhesion kinase and matrix metalloproteinase-2 in HEp-2 cells. Mol Cell Biochem 357: 95-106, 2011.

15. Rao Q, Chen Y, Yeh CR, Ding J, Li L, Chang C and Yeh S: Recruited mast cells in the tumor microenvironment enhance bladder cancer metastasis via modulation of ER $\beta / C C L 2 / C C R 2$ EMT/MMP9 signals. Oncotarget 7: 7842-7855, 2016.

16. Qin Y, Tang B, Hu CJ, Xiao YF, Xie R, Yong X, Wu YY, Dong H and Yang SM: An hTERT/ZEB1 complex directly regulates E-cadherin to promote epithelial-to-mesenchymal transition (EMT) in colorectal cancer. Oncotarget 7: 351-631, 2016.

17. Zheng X, Carstens JL, Kim J, Scheible M, Kaye J, Sugimoto H, Wu CC, LeBleu VS and Kalluri R: Epithelial-to-mesenchymal transition is dispensable for metastasis but induces chemoresistance in pancreatic cancer. Nature 527: 525-530, 2015.

18. Burnett JP, Korkaya H, Ouzounova MD, Jiang H, Conley SJ, Newman BW, Sun L, Connarn JN, Chen CS, Zhang N, et al: Trastuzumab resistance induces EMT to transform HER $2^{+}$ PTEN $^{-}$to a triple negative breast cancer that requires unique treatment options. Sci Rep 5: 15821, 2015.

19. Attramadal CG, Kumar S, Boysen ME, Dhakal HP, Nesland JM and Bryne M: Tumor budding, EMT and cancer stem cells in T1-2/N0 oral squamous cell carcinomas. Anticancer Res 35: 6111-6120, 2015

20. Wang SC, Chai DS, Chen CB, Wang ZY and Wang L: HPIP promotes thyroid cancer cell growth, migration and EMT through activating PI3K/AKT signaling pathway. Biomed Pharmacother 75: 33-39, 2015.

21. Buczek ME, Miles AK, Green W, Johnson C, Boocock DJ, Pockley AG, Rees RC, Hulman G, van Schalkwyk G, Parkinson R, et al: Cytoplasmic PML promotes TGF- $\beta$-associated epithelialmesenchymal transition and invasion in prostate cancer. Oncogene 43: 124-127, 2015.

22. Ota I, Masui T, Kurihara M, Yook JI, Mikami S, Kimura T, Shimada K, Konishi N, Yane K, Yamanaka T, et al: Snail-induced EMT promotes cancer stem cell-like properties in head and neck cancer cells. Oncol Rep 35: 261-266, 2016.

23. Broster SA and Kyprianou N: Epithelial-mesenchymal transition in prostatic disease. Future Oncol 11: 3197-3206, 2015.

24. Lee SC, Kim OH, Lee SK and Kim SJ: IWR-1 inhibits epithelialmesenchymal transition of colorectal cancer cells through suppressing Wnt/ $\beta$-catenin signaling as well as survivin expression. Oncotarget 6: 27146-27159, 2015.

25. Thiery JP: Epithelial-mesenchymal transitions in tumour progression. Nat Rev Cancer 2: 442-454, 2002.

26. Thiery JP, Acloque H, Huang RY and Nieto MA: Epithelialmesenchymal transitions in development and disease. Cell 139: 871-890, 2009. 
27. D'Assoro AB, Liu T, Quatraro C, Amato A, Opyrchal M, Leontovich A, Ikeda Y, Ohmine S, Lingle W, Suman V, et al: The mitotic kinase Aurora-A promotes distant metastases by inducing epithelial-to-mesenchymal transition in $\mathrm{ER} \alpha^{+}$breast cancer cells. Oncogene 33: 599-610, 2014.

28. Guan Z, Wang XR, Zhu XF, Huang XF, Xu J, Wang LH, Wan XB, Long ZJ, Liu JN, Feng GK, et al: Aurora-A, a negative prognostic marker, increases migration and decreases radiosensitivity in cancer cells. Cancer Res 67: 10436-10444, 2007.

29. Thiyagarajan V, Tsai MJ and Weng CF: Antroquinonol targets FAK-signaling pathway suppressed cell migration, invasion, and tumor growth of C6 glioma. PLoS One 10: e0141285, 2015.

30. Sun L, Liu L, Liu X, Wang Y, Li M, Yao L, Yang J, Ji G, Guo C, Pan Y, et al: MGrl-Ag/37LRP induces cell adhesion-mediated drug resistance through FAK/PI3K and MAPK pathway in gastric cancer. Cancer Sci 105: 651-659, 2014.

31. Lin Y, Rao J, Zha XL and Xu H: Angiopoietin-like 3 induces podocyte F-actin rearrangement through integrin $\alpha_{\mathrm{v}} / \beta_{3} / \mathrm{FAK} /$ PI3K pathway-mediated Rac1 activation. Biomed Res Int 2013: 135608, 2013.

32. Fu QF, Liu Y, Fan Y, Hua SN, Qu HY, Dong SW, Li RL, Zhao MY, Zhen Y, Yu XL, et al: Alpha-enolase promotes cell glycolysis, growth, migration, and invasion in non-small cell lung cancer through FAK-mediated PI3K/AKT pathway. J Hematol Oncol 8: $22,2015$.

33. Hochwald SN, Nyberg C, Zheng M,Zheng D, Wood C, MassollNA, Magis A, Ostrov D, Cance WG and Golubovskaya VM: A novel small molecule inhibitor of FAK decreases growth of human pancreatic cancer. Cell Cycle 8: 2435-2443, 2009.
34. Greger JG, Eastman SD, Zhang V, Bleam MR, Hughes AM, Smitheman KN, Dickerson SH, Laquerre SG, Liu L and Gilmer TM: Combinations of BRAF, MEK, and PI3K/mTOR inhibitors overcome acquired resistance to the BRAF inhibitor GSK 2118436 dabrafenib, mediated by NRAS or MEK mutations. Mol Cancer Ther 11: 909-920, 2012.

35. Evans EB and Lin SY: New insights into tumor dormancy: Targeting DNA repair pathways. World J Clin Oncol 6: 80-88, 2015.

36. Niu NK, Wang ZL, Pan ST, Ding HQ, Au GH, He ZX, Zhou ZW, Xiao G, Yang YX, Zhang X, et al: Pro-apoptotic and pro-autophagic effects of the Aurora kinase A inhibitor alisertib (MLN8237) on human osteosarcoma U-2 OS and MG-63 cells through the activation of mitochondria-mediated pathway and inhibition of $\mathrm{p} 38 \mathrm{MAPK} / \mathrm{PI} 3 \mathrm{~K} / \mathrm{Akt} / \mathrm{mTOR}$ signaling pathway. Drug Des Devel Ther 9: 1555-1584, 2015.

37. Dar AA, Goff LW, Majid S, Berlin J and El-Rifai W: Aurora kinase inhibitors - rising stars in cancer therapeutics? Mol Cancer Ther 9: 268-278, 2010.

38. Gu K,Li MM, Shen J,Liu F, Cao JY, Jin S and Yu Y: Interleukin-17induced EMT promotes lung cancer cell migration and invasion via NF-kB/ZEB1 signal pathway. Am J Cancer Res 5: 1169-1179, 2015. 